\title{
Selektive Wahrnehmung des BAG
}

\section{Beat Bumbacher}

Leiter Tarifdienst der FMH
Korrespondenz:

Dr. med., RA. lic. iur. Beat Bumbacher Tarifdienst FMH

Gösgenstrasse 8

CH-4600 Olten

Tel. 0622879696

Fax 0622879690

beat.bumbacher@fmh.ch
Der Artikel «Laboranalysen in der ärztlichen Praxis», der im BAG-Bulletin 12/09 erschienen ist, kann nicht einfach so hingenommen werden. Bereits in der Einleitung wird darauf hingewiesen, dass das Tarifmodell der jetzt noch gültigen Analysenliste (AL) veraltet sei und dass die nun neue ganze AL auf den aktuellen Gegebenheiten betriebswirtschaftlich bemessen wurde. Es sollte mittlerweile wohl jedem gesundheitspolitisch Interessierten im Lande klar geworden sein, dass dem nicht so ist. Für das Praxislabor wurden keine spezifischen Berechnungsmodelle, geschweige denn Berechnungen erstellt, und das Resultat ist deshalb betriebswirtschaftlich weder im Modell noch im Resultat schlüssig. Die Konsequenzen daraus wurden in den letzten Tagen immer wieder erläutert: Der Eingriff in die AL ist ein strukturpolitischer Akt, der sich beim unternehmerisch denkenden Arzt (auch dies eine Forderung von Politik und Kassen) in einer Anpassung seiner Tätigkeit bemerkbar macht. Wir gehen davon aus, dass sich rund zwei Drittel der Praxislaboratorien wegen Mindereinnahmen nicht mehr rechnen und mittelfristig gefährdet sind. Das BAG wird das vom KVG vorgegebene Ziel verfehlen, eine effiziente Versorgung mit Analyseleistungen zu gewährleisten.

\section{Anleitung zur Rosinenpickerei}

Interessant wird der Artikel aber dann, wenn sich das BAG gegen den Vorwurf wehrt, dass mit der neuen AL weniger verdient werden könnte. Gezielt werden verschiedene Einzelbestimmungen herausgepickt und die Vergütungen heute und unter der neuen AL verglichen. Als Einzelbestimmungen kann diesen Zahlen nichts entgegengehalten werden, die Preise stimmen für Grosslabors. Hingegen macht es kaum Sinn, Einzelbestimmungen ohne einen medizinischen Kontext anzuschauen. Um auch diesen Aspekt $\mathrm{zu}$ kontern, gibt nun das BAG vier typische Laborkombinationen an, nämlich für akute Gastritis (Hb/Lc, CRP), Arthritis (CRP, Urat, Lc-Diff), afebrile Allgemeinerkrankungen (CRP, Hämatogramm I, Lc-Diff) und chronische Herzinsuffizienz (Kreatinin, Kalium). Man müsste bei diesen Beispielen fast sagen: Wer sucht, der findet. Relativ einfach lassen sich Beispiele finden, für die nur zwei bis drei Analysen nicht ausreichen. Vielfach kommen zur sorgfältigen Abschätzung der medizinischen Situation beim häufigen Fall von nicht isolierten Krankheitsbildern noch ein bis zwei Chemiewerte hinzu, und dann sieht die Rechnung schon völlig anders aus. Die gewählten Beispiele des BAG sind insofern nicht reprä- sentativ, sondern es resultiert nur deshalb kein Verlust gegenüber heute, weil es einen Verdünnungseffekt der Zuschläge von Präsenztaxe und weiteren Zuschlägen gibt, der mit einer zunehmenden Zahl von Analysen ebenfalls zunimmt. Oder einfach gesagt: Wer mehr als drei Analysen pro Sitzung durchführt, wird gegenüber der Vergütungssituation von heute mit grosser Sicherheit schlechter fahren. In diesem Sinne hat sich auch der BAG-Vizedirektor Peter Indra anlässlich des Mediengesprächs vom 9. März geäussert. Letztlich hat das nicht mehr viel mit den realen Fragestellungen in der Praxis zu tun, sondern ist Anleitung zur Rosinenpickerei. Das BAG lehnte sich mit diesen Guidelines für die medizinische Praxis sehr weit aus dem Fenster, und es würde uns nicht erstaunen, wenn diese sogar für «nurse practitioners» verfasst worden wären ...

\section{Falschinformationen}

Daneben hat es im Artikel aber auch Falschinformationen. So wird etwa behauptet, dass in der AL nur eine Liste geführt werden könnte. Abgesehen davon, dass bereits heute eine Laborbestimmung für das Praxis- und das Grosslabor unterschiedlich gerechnet und abgebildet wurde, hat ein Rechtsgutachten der FMH gezeigt, dass sehr wohl auch verschiedene Modelle in diese AL einfliessen können, wenn dadurch die Abgeltung spezifischer berechnet werden kann. Das Point-of-care-Modell hätte gut übernommen werden können. Es muss hier allerdings angefügt werden, dass die FMH ihr Modell immer als Diskussionsgrundlage gesehen hat, um dem BAG aufzuzeigen, wie das Praxislabor sachgerecht und betriebswirtschaftlich gerechnet werden könnte. Aber das BAG wollte den Faden nicht aufnehmen und hat sich dieser Diskussion nicht gestellt. Der nun erhobene Vorwurf, das Modell hätte Mehrkosten zur Folge, muss deshalb ganz entschieden von der Hand gewiesen werden. Das Modell hätte nämlich sehr gut so gesteuert werden können, dass solche Mehrkosten eben gerade nicht angefallen wären. Im Übrigen ist es auch falsch, wenn das BAG behauptet, dass gewisse Kosten wie Empfang, Wartezimmer usw. gleich mehrfach erstattet würden. Es zeigt sich gerade an diesem Detail, dass das BAG sowohl den TARMED als auch das Point-of-care-Modell nicht genügend studiert hat. Es ist leider zu befürchten, dass sich gerade aufgrund dieses Faktums das BAG nicht in die Karten schauen lassen wollte und den Erarbeitungsprozess bis heute nicht transparent gemacht hat. 